\title{
Tuberous Sclerosis Complex : A Case Report
}

\author{
Shahara Haque ${ }^{1 *}$ \\ Tarannum Morshed ${ }^{2}$ \\ Hasina Begum ${ }^{\prime}$ \\ Shahidul Islam ${ }^{1}$ \\ Kaniz Fatema' \\ Afroza Begum \\ Muhammad Shoyab
}

'Department of Radiology \& Imaging

Dhaka Medical College

Dhaka, Bangladesh.

${ }^{2}$ Department of Radiology \& Imaging Bangladesh Institute for Research \& Rehabilitation in Diabetes

Endocrine \& Matabolic Diseases (BIRDEM) Dhaka, Bangladesh.
${ }^{*}$ Correspondence to:

\section{Dr. Shahara Haque} Associate Professor

Department of Radiology \& Imaging

Dhaka Medical College

Dhaka, Bangladesh.

Mobile : +8801818464627

E-mail : awwal70@yahoo.com

w w w. c m o s h m cj.org

\begin{abstract}
The aim of this report is to present various clinical and radiological features of a young female patient with tuberous sclerosis who exhibited multiple hamartomas of various organ system. Tuberous sclerosis is a rare neurocuteneous syndrome exhibiting multiple hamartomatous proliferations that may involve multiple organ system such as brain, kidney, heart, lungs, eyes and skin. An 18 year old female patient presented with abdominal pain and swelling. Clinical examination of the patient revealed presence of facial angiofibromas and huge left flank mass. She also gave history of twin pregnancy with IUD. USG of abdomen showed bilateral gross angiomyolipoma with necrosis and haemorrhage in left kidney. CT and MRI of brain showed presence of multiple cortical tubers and calcified subependymal nodules. This case report is a good example of complex nature of tuberous sclerosis. The diagnosis and management of these patients depend on the presentation of the disease.
\end{abstract}

Key words: Tuberous sclerosis complex; Renal angiomyolipoma; Cortical tubers; Subependymal nodules.

\section{INTRODUCTION}

Tuberous sclerosis is a rare syndrome with an estimated incidence of 1 in 6000 to 1 in 10,000 live births. The name tuberous sclerosis is derived from the characteristic tuber like growth occurring in the brain which calcifies with age and become sclerotic. The disorder was once known as epiloia or Bournville's disease and was first identified by French physician Bournville in 1880 .

There is family history of disease in $50 \%$ of affected patients with a autosomal mode of inheritance. Some individual acquire tuberous sclerosis complex through a process called gonadal mosaicism. TSC is caused by defects or mutations in two genes- TSC1 and TSC-2. Only one of the genes needs to be affected to produce the disease. The TSC-1 gene is located on chromosome 9 (9q34) and produces protein called hamartin. The TSC-2 gene is on chromosome 16 (16p 13.3) and produces tuberin.

\section{CASE REPORT}

An 18 years old female patient reported to the OPD of medicine Department of DMCH. Dhaka, Bangladesh with the complaints of abdominal pain and distension. The patient had multiple brown lesions over her face. There was no history of bleeding, itching, pain or change in the size of the lesions. None of her family members suffer from any similar condition.

Clinical examination revealed multiple angiofibromas appearing as well defined roughly round to oval dark brown firm papules all over her face. A shageern patch (hyperpigmented plaque) was present over left side of her face (Fig. 1). She also had huge mass on left hypochondriac region. 
An USG of abdomen revealed multiple fatty depositions in right kidney. The left kidney was replaced by inhomogeneous mass with fat and necrotic changes. Both pelvicaleceal system could not be well delineated (angiomyolipoma with necrosis and haemorrhage).

CT scan of brain showed multiple calcified subependymal nodules and cortical tubers (Fig. 2).

CT scan of abdomen also detected the angiomyolipoma in left kidney.

Findings of MRI of brain was

Thickened cortex with focal areas of predominantly FLAIR hyperintensity and T1WI isointensity and T2WI hyperintensity are noted in both frontal, parietal and occipital lobes with gyral swelling (Fig. 3,4).

Tiny subependymal nodules are seen in both lateral ventricle which appears isointense on T1WI and Hypointense on T2WI. Final MR diagnosis was Multiple cortical tubers and calcified subependymal nodules- Suggestive of tuberous sclerosis.

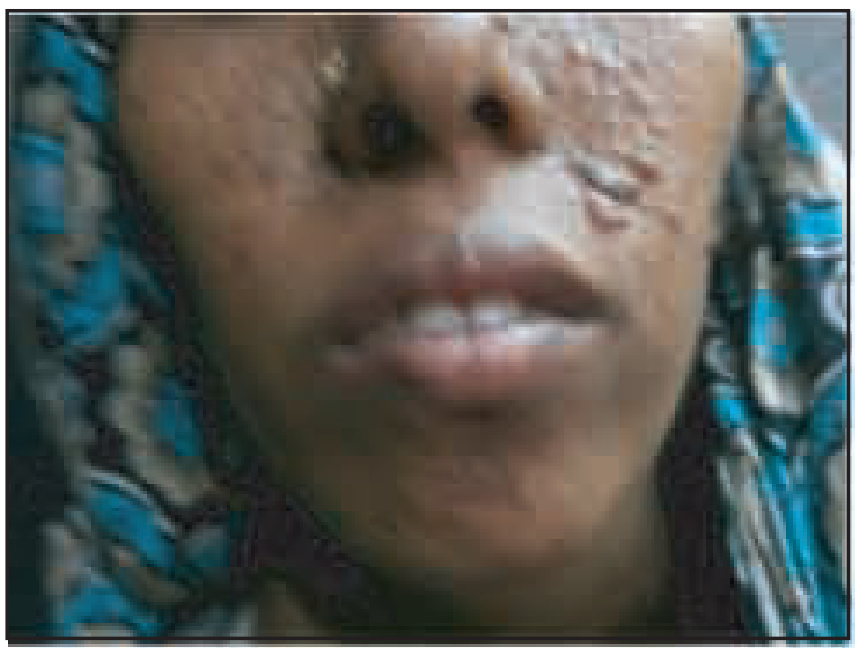

Figure 1 : Shageern patch (hyperpigmented plaque)

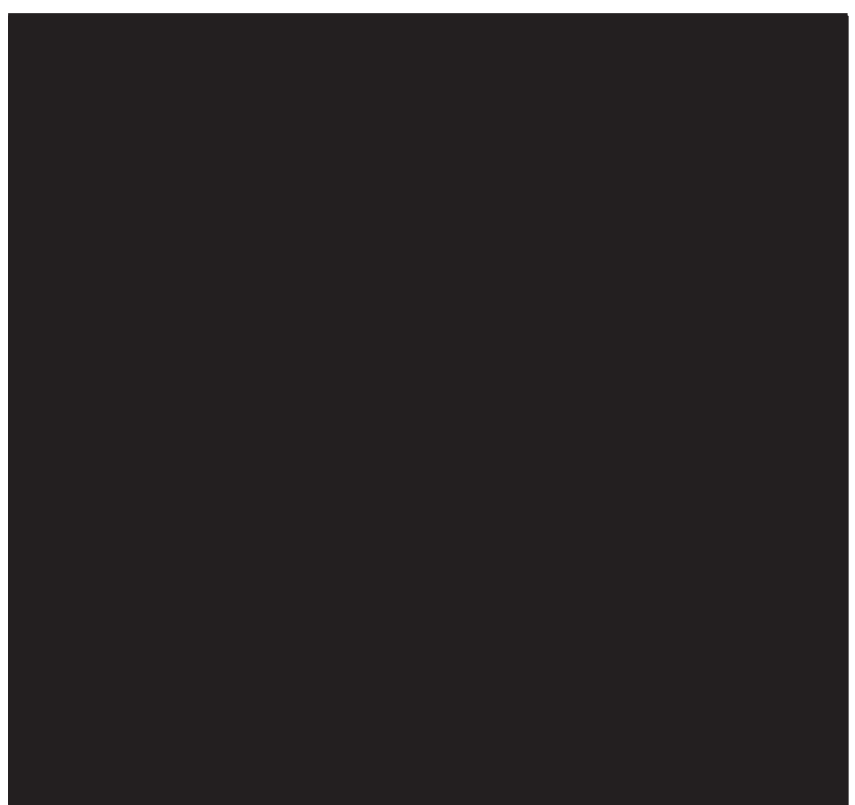

Figure 2 : CT scan of brain showing multiple calcified subependymal nodules

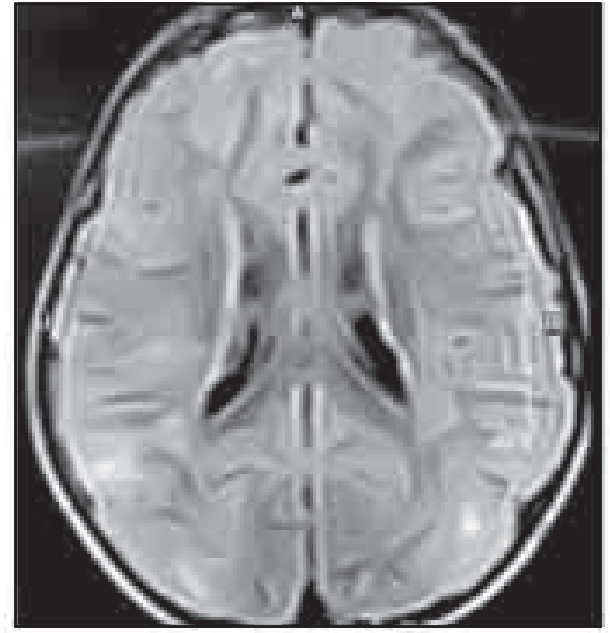

Figure 3 : T1W MR images of brain showing multiple cortical tubers and calcified subependymal nodules

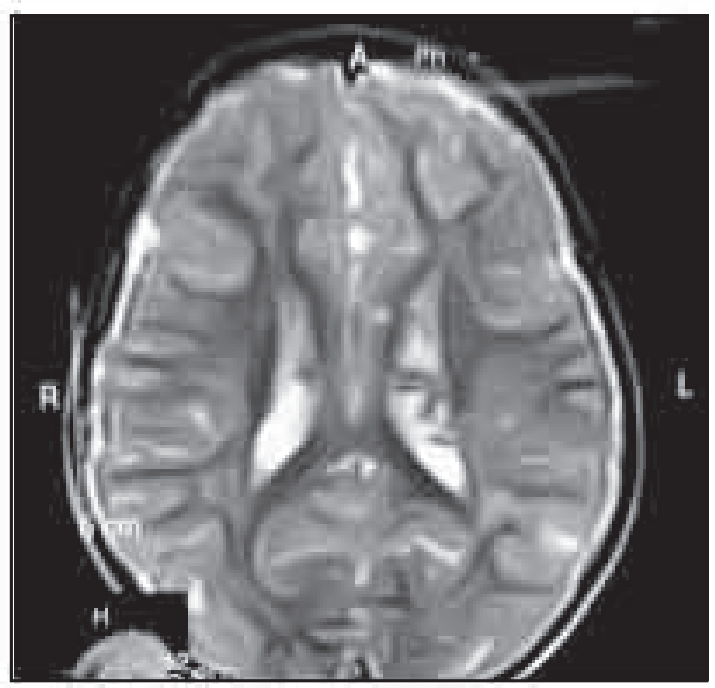

Figure 4 : T2W MR images of brain showing multiple cortical tubers and calcified subependymal nodules

\section{DISCUSSION}

Manifestations of tuberous sclerosis can become apparent in persons of any age, but most patients have clinical symptoms before they are aged 10 years. The disease develops as an abnormal growth of ectodermic cells producing tumors extending to areas of the head, heart, brain, eyes, skin and kidneys ${ }^{1}$.

In 2012, the International Tuberous Sclerosis Complex Consensus Conference published new diagnostic criteria for diagnosis of tuberous sclerosis.

\section{Clinical diagnostic criteria}

\section{Major features}

1. Hypomelanotic macules (3, at least 5-mm diameter)

2. Angiofibromas (3) or fibrous cephalic plaque

3. Ungual fibromas (2)

4. Shagreen patch 
5. Multiple retinal hamartomas

6. Cortical dysplasias*

7. Subependymal nodules

8. Subependymal giant cell astrocytoma

9. Cardiac rhabdomyoma

10. Lymphangioleiomyomatosis (LAM)y

11. Angiomyolipomas (2)y

\section{Minor features}

1. "Confetti" skin lesions

2. Dental enamel pits $(>3)$

3. Intraoral fibromas (2)

4. Retinal achromic patch

5. Multiple renal cysts

6. Nonrenal hamartomas

Definite diagnosis: Two major features or one major feature with 2 minor features

Possible diagnosis: Either one major feature or 2 minor features According to the criteria the patient reported had more than 2 major criteria and was considered as TSC.

There are many cutaneous stigmata of TSC and they are seen in almost all patients with the disorder. The most common and earliest skin finding in TSC is multiple hypopigmented macules (also called ashleaf spots). Adenoma sebaceum is a hamartoma composed of connective and vascular elements and is properly termed an angiofibroma they are pathognomonic of TSC. Angiofibromas form discrete pink papules on the malar region of the face in $70 \%$ of TSC patients ${ }^{2,3}$.

Patients with tuberous sclerosis complex (TSC) can develop a number of renal lesions, the most common being angiomyolipomas and cysts ${ }^{4}$. Renal cysts are rarely symptomatic in patients with TSC, unless the patient exhibits the polycystic kidney variety of TSC. It has been reported that up to $80 \%$ of adult patients with TSC will develop angiomyolipoma and that these lesions tend to increase in size over time

The intracranial features of TSC are cortical or subcortical tubers, subependymal nodules, subependymal giant cell astrocytomas, and white matter radial migration lines 5 . Tubers are most commonly found in the cerebrum, $90 \%$ being present in the frontal lobes. On NECT in early stages tubers appear as low density subcortical masses. In late stages the appear isodense to barin. $50 \%$ calcify by the age of 10 years. On T2weighted and FLAIR MR images, tubers typically appear as areas of increased signal intensity in the cortical and subcortical regions. Tubers exhibit contrast enhancement in approximately $3-4 \%$ of cases. Ninety-five percent of tubers are multiple, but in rare instances solitary cortical tubers are seen. Less commonly tubers are present in the cerebellum. Tubers rarely are found in the brainstem and spinal cord.
Subependymal nodules (SEN) are found on the walls of the lateral ventricles and are either discrete or roughly confluent areas of rounded hypertrophic tissue. Typically benign, sub ependymal nodules can degenerate into sub ependymal giant cell astrocytomas in $5-10 \%$ of cases. On NECT the nodules occur anywhere along the ventricular surface but are most commonly found at $t$ he caudothalamic groove in the region of the foramen of Monro. 50\% calcify. Enhancing SEN may be suspicious for sub ependymal giant cell astrocytoma.

Microcephaly may be found in patients with TSC. The cerebral gray and white matter volumes are lower than those of agematched controls ${ }^{6}$.

Pulmonary lymphangioleiomyomatosis probably affects $1-3 \%$ of patients with tuberous sclerosis. Although some articles report the occurrence of LAM in 1 to $3 \%$ of the patients with TS.

A cardiac rhabdomyoma can be discoverded using echocardiography in approximately $50 \%$ of TSC patients. However, the incidence in the newborn may be as high as $90 \%$ and in adults as low as $20 \%$. Rhabdomyomas have been diagnosed by two-dimensional echocardiography in the fetus ${ }^{7}$.

Ophthalmic features associated with TSC can be divided into retinal and non-retinal. The retinal lesions are known as astrocytic hamartomas ${ }^{8}$. Non retinal lesions include coloboma, angoifibroma of the eyelid and papilledema (related to hydrocephalus).

Multiple bony changes have been described in TSC of which sclerotic lesions are the most common. Hyperostosis of inner table of skull, periosteal new bone formation, scoliosis, and bone cysts have also been described ${ }^{9}$.

Patients with Tuberous sclerosis complex (TSC) range from intellectually normal to severely mentally retarded. TSC is often associated with mental retardation (in $70 \%$ of cases) and epilepsy (90\%). Seizures are the most common neurologic symptom of TSC occurring in $92 \%$ of patients ${ }^{10}$.

Prognosis of the disease depends on the severity or multiplicity of organ involvement.

\section{CONCLUSION}

It is not uncommon for patients with TSC to have symptoms or signs that do not lead to immediate diagnosis. In some cases, diagnosis is delayed for prolonged periods of time. Early diagnosis is very important. Radiology plays the key role in diagnosis and detecting complex nature of the disease. Thorough clinical and radiological evaluation, continuous monitoring of symptoms, family planning, and genetic counseling can reduce the morbidity and mortality rate.

\section{DISCLOSURE}

All the authors declared no competing interest. 


\section{REFERENCES}

1. Gupta S, Bhowate R, Degwekar SS. Clinical and Radiological Findings Related to Tuberous sclerosis Complex: A case report. J Contemp dent Prac. 2008;(9)4:085-091

2. Harper JJ, Trembath RC,. Genetics and Genodermatosis in : breathnach, Neil Cox, Christopher Griffiths. Rook's Textbook of Dermatology, 7th edition, volume-1Blackwell, Australia. 2004; 1233-1237.

3. Henry J, Baskin Jr. The pathogenesis and imaging of the tuberous sclerosis complex. Pediatr Radiol. 2008; 38: 936-952.

4. Keith A, Casper MS, Lane FD, BinChen, John JB. Tuberous Sclerosis Complex: Renal Imaging Findings. Radiology. 2002; 225.

5. Babak NK and Noriko S. Neuroimaging of Tuberous Sclerosis: spectrum of Pathologic Findings and Frontiers in Imaging. American Journal of Roentgenology. 2008; 190(5): 304-309.

6. Flavia GV, Edson M, Glaucia Z. et al. tuberous sclerosis with pulmonary lymphangioleiomatosis and renal angiomyolipomas. Computed tomographic findings: a case report. Cases Journal. 2009;2:9124.

7. Maryum M, Mohammad D, Mahmood M. Prenatal Diagnosis of Tuberous Sclerosis by Fetal Echocardiography with an Unusual Clinical course After Birth. arch cardiovasc Image. 2013; 1(2):83-85.

8. Rowley SA, O'Callaghan FJ, Osborne JP. Ophthalmic manifestations of tuberous sclerosis: a population based study. Br j Ophthalol. 2001; 85: 420-423.

9. Rupa R, Sadhna V. Clinically relevant Imaging in tuberous Sclerosis. Journal of Clinical imaging Science. 2011; 1: 39.

10. Shobha B., Ashwini R, Veerendra SP, Sunil B. Tuberous Sclerosis Complex Syndrome A Rare Case Report. Journal of Medical Education \& Research. 3(1):57-63. 